\title{
Tagungsbericht
}

Torsten Seela, Leipzig

\section{Auf der Suche nach dem Königsweg}

\section{Jubiläumskolloquium „100 Jahre bibliothekarisches Studium in Leipzig“}

DOI 10.1515/iwp-2015-0003

In der Regel wird das Jubiläum einer Institution genutzt, um Rückschau zu halten, Bilanz zu ziehen, Erreichtes zu feiern. Das ist gewiss gut und berechtigt. Dass man einen solchen Jahrestag aber auch anders begehen kann, war die Idee des aus Anlass des hundertjährigen Bestehens Leipziger bibliothekarischer Ausbildungs- und Studiengänge am 15. Oktober 2014 veranstalteten Kolloquiums.

Den Organisatoren der Veranstaltung an der Fakultät Medien der Hochschule für Technik, Wirtschaft und Kultur (HTWK) Leipzig war daran gelegen, einen Blick in die nähere Zukunft der Bibliothekspraxis $\mathrm{zu}$ werfen, nicht zuletzt, um den Studiengang Bibliotheks- und Informationswissenschaft den zu erwartenden neuen Anforderungen anzupassen. Und so begaben sie sich in der Vorbereitung des Kolloquiums ,auf die Suche nach dem Königsweg im 21. Jahrhundert“, mussten aber sehr bald feststellen, dass es dem übertragenen Sinne des Wortes nach, anders als beim historischen Königsweg von Ephesos nach Susa, mehrere Wege geben könne. Drei davon wurden in der Veranstaltung thematisiert, um deren Königsweg-Potential auszuloten.

Dem Vormittag gehörte dabei eine Podiumsdiskussion zum Thema Kreuzqualifikationen für das Bibliothekswesen. Mit der Etablierung eines Studienschwerpunktes Bibliothekspädagogik im Masterstudiengang Bibliotheksund Informationswissenschaft und mit der Begründung eines ganz neuen Studienangebots Bibliotheksinformatik, von der Praxis nachhaltig begrüßt, hatte sich die HTWK schon vor dem Jubiläum auf diesen Königsweg begeben, ahnend, dass derartige Qualifikationen in den Bibliotheken immer stärker nachgefragt werden dürften.

Prof. Dr. Klaus Tochtermann (Deutsche Zentralbibliothek für Wirtschaftswissenschaften), Prof. Dr. Michael Frank (HTWK Leipzig), Bettina HarLING (Stadtbibliothek Mannheim) und Prof. Dr. Arend Flemming (Städtische Bibliotheken Dresden) bestätigten aus eigener beruflicher Erfahrung den Bedarf an kreuzqualifizierten Mitarbeitern, weil den Herausforderungen der Moderne nicht mehr allein mit dem klassischen bibliothekarischen „Handwerk“ begegnet werden könne. Doch wenn sich auch, wie ТоснTERMANN ausführte, neue Distributionskanäle für Katalogdaten auftun, die Zahl der Ausleihen gegenüber den Downloads dramatisch sinke, die Retrodigitalisierung die Massenentsäuerung verdränge - gänzlich ohne bibliothekarische Kenntnisse seien diese Probleme nicht $\mathrm{zu}$ bewältigen, und schon gar nicht, betonte FLEMming, ohne die bibliothekarisch-ideelle Grundmotivation, die die Kundenzufriedenheit in den Mittelpunkt stelle.

Vergleichbares gelte auch für die Bibliothekspädagogik, wie HarLing berichtete. Das Streben nach Bildungsgerechtigkeit, die daraus erwachsende Notwendigkeit von Sprach- und Leseförderung sowie der Entwicklung von Informations- und Medienkompetenz stelle die Mitarbeiter vor deutlich größere Aufgaben, zumal das pädagogische Studium in der Regel keinerlei bibliothekarische Kenntnisse vermittle.

Einig waren sich die Diskussionsteilnehmer, dass es nicht darum gehen könne, bibliothekarische und fachspezifische Kompetenzen gegeneinander auszuspielen, dass vielmehr der Bibliothekar mit Spezialkompetenzen genauso erforderlich sei wie der Fachspezialist mit bibliothekarischen Kenntnissen, ohne die es nicht gehe, wie FRANk herausstellte, denn die Begriffswelt der Bibliotheken und ihre Kultur müssten gelernt werden, um angemessen wirken zu können, und der Bibliothekar wiederum müsse dem Spezialisten mit Gesprächsverständnis zur Seite treten.

Mithin ergab die Debatte ein rückhaltloses Ja zum Erwerb von Kreuzqualifikationen; offen blieb allerdings die Frage nach dem Weg dorthin - konsekutiv oder integrativ? Oder hat beides seine Berechtigung?

Der lebhaften Diskussion vom Vormittag folgten am Nachmittag Referate zu den Themenblöcken „Cloud-Computing“ und „Reorganisation“. Zu ersterem berichtete Dr. Uwe Risch (HeBIS-Verbundzentrale) über Stand und Perspektiven des CIB-Projekts, das seit dem 1. 9. 2013 vorangetrieben wird, und in dem KOBV, BVB und HeBIS kooperieren. Der Referent stellte dabei Anforderungen und Chancen einer reformierten Zentralkatalogisierung in den Mittelpunkt seiner Ausführungen: durch die Internationali- 
sierung käme den Bibliotheken erhöhte Bedeutung als Partner für Bildung, Forschung und Lehre zu, es könnten aber auch die durch Rationalisierung frei werdende Mittel neu investiert werden. Gleichzeitig erfordere jedoch die plattformübergreifende Erfassung bibliothekarischer Metadaten nach internationalen Standards von den mit dieser Aufgabe betrauten Bibliotheksmitarbeitern erhöhte Aufmerksamkeit. Sorgen hinsichtlich der Datensicherheit in der Cloud zerstreute er mit dem Hinweis auf Einrichtung eines nationalen Datenfensters. Risch sah auch keineswegs ein Ende der Verbünde voraus, sondern vertrat die Auffassung, dass ihnen im Rahmen einer neuen Arbeitsteilung die intensivierte Betreuung von Bibliotheksfunktionen zukäme.

Reinhard AltenHÖNER (Deutsche Nationalbibliothek) und Prof. Dr. Andreas Degkwitz (UB der Humboldt-Universität zu Berlin) stellten in ihren aus der Praxis geschöpften Berichten Vorteile, aber auch Risiken der Cloud-basierten Katalogisierung heraus. Der leichtere Zugang zu internationalen Datenangeboten, so DeGKowitz, und die Ablösung der regionalen Verbunddatenbanken seien unabweisbare Vorteile, aber auch die höhere Wirtschaftlichkeit durch Auslagerung der System-Basis und die Standardisierung des Workflows. Altenhöner wies dagegen darauf hin, dass die Einsparungseffekte durchaus noch nicht belegt seien. Rückhaltlos legte DeGKowitz aber auch die Gefahren offen, die von den neuen Entwicklungen ausgingen. Sie seien hier nur stichwortartig genannt: mögliche Qualitätseinbußen, geringe Berücksichtigung lokaler Besonderheiten, Abhängigkeit vom Betreiber der Cloud mit unabsehbaren Folgen für die Kostenentwicklung; nicht zuletzt erforderten auch alle Fragen, die mit Datenhoheit, Datenschutz und Datensicherheit zusammenhingen, weitere Klärung.

Mit Bezug auf Winston Churchills Lobpreis des Wandels eröffnete Michael GoLSCH (SLUB Dresden) den dritten Themenblock, der sich mit der notwendig raschen Veränderung bestehender Bibliotheksstrukturen befasste.

Ausgehend von der Verlagerung der konventionellen Literaturversorgung hin zur digitalen, verwies er auf die Konsequenzen, die sich aus diesem Wandel ergeben: die Arbeit ändere sich und werde komplexer, die Arbeit werde jedoch auch weniger, so dass sich höhere Anforderungen an das zahlenmäßig geringer werdende Personal ergäben. Instrument, diese Veränderung zu gestalten, sei das Change Management, für das die Geschäftsführung den Impuls zu geben habe, und das durch die Beschäftigten via Aufgabenkritik, Produktkritik und Strukturkritik getragen würde. Die Strukturveränderungen seien jedoch nur dann realisierbar, wenn durch proaktives und zugleich partizipatives Handeln der Beteiligten ausreichend Transparenz hergestellt würde.
Elke BEER (Stadtbibliothek Chemnitz) berichtet dazu aus der Praxis, wie an der Stadtbibliothek Chemnitz seit 2002 Eigeninitiative durch Teambildung und jährliche Zielvereinbarungen mit Controlling gefördert wurden. Sie wies aber auch auf die Verantwortung der Bibliotheksleitung hin, diesen Veränderungsprozess angesichts der immer älter werdenden Belegschaft sensibel und mit menschlichem Verständnis zu steuern.

Prof. Dr. Hans-Christoph Новонм (FH Potsdam) erläuterte schließlich, welche Folgen der Wandel der Bibliothekspraxis für die Gestaltung der Curricula hat. Die permanent stattfindende Berufsbilddiskussion habe in Potsdam zur Abschaffung traditioneller Lehrinhalte (Bibliotheksgeschichte, Literaturkunde etc.) geführt, um neue Lehrinhalte (Bibliothekstechnik, Metadaten, Management) in ausreichendem Maße in der Lehre etablieren zu können. Dabei käme es darauf an, systemische und instrumentelle Kompetenzen $\mathrm{zu}$ vermitteln und $\mathrm{zu}$ entwickeln. Studentische Projekte und Graduierungsarbeiten seien entsprechend inhaltlich ausgerichtet und trügen ganz im Sinne der von R. David Lankes geforderten „new librarianship“ zu einer letztlich evidenzbasierten bibliothekarischen Praxis bei.

Angesichts der Fülle des Programms blieb am Ende leider wenig Zeit zu einer intensiven Aussprache. Doch der optimistische Grundtenor aller Beiträger wird dem Publikum gewiss im Gedächtnis bleiben. So begnügt sich der Berichterstatter als Fazit mit einem Zitat, abermals von Winston Churchill: „There is nothing wrong with change, if it is in the right direction!“

Darauf bauend, so ist der Autor dieser Zeilen überzeugt, wird der Studiengang Bibliotheks- und Informationswissenschaft der HTWK Leipzig die Zukunft der bibliothekarischen Ausbildung bis hin zum nächsten Jubiläum aktiv mitgestalten.

Deskriptoren: Bibliothekswesen, Tagung, Hochschulausbildung, HTWK Leipzig

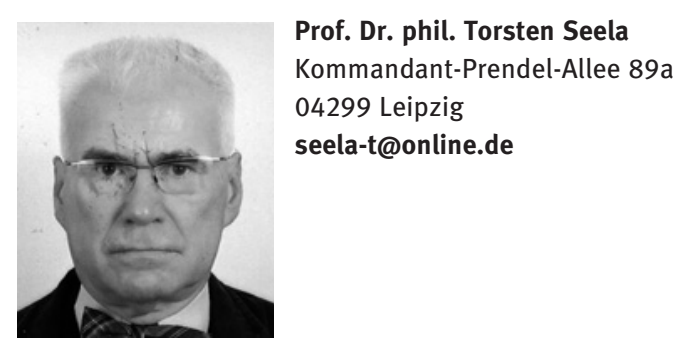

Prof. Dr. phil. Torsten Seela (geb. 1948) war von 1992 bis 2013 Professor am Studiengang Bibliotheks- und Informationswissenschaft der HTWK Leipzig; 1995/97 und 2000/02 Dekan des seinerzeitigen Fachbereichs Buch und Museum. 